\title{
Patient-reported outcome measures after routine periodontal and implant surgical procedures
}

Tan, Wah Ching ; Krishnaswamy, Gita ; Ong, Marianne M A ; Lang, Niklaus P

\begin{abstract}
OBJECTIVES: To compare patient-reported outcome measures (PROMs) after different dental surgical procedures over a 1-week post-surgical period and in relation to duration of the surgery, and periosteal releasing incisions. To evaluate the prevalence of post-surgical complications. MATERIAL METHODS: Four hundred and sixty-eight healthy dental patients requiring surgeries, such as crown lengthening (CL), open flap debridement (OFD) and implant installation (IMP) in the National Dental Centre, Singapore (2009-2011), were consecutively recruited. PROMs on bleeding, swelling, pain and bruising were obtained using Visual Analogue Scales (VAS) on days $0,3,5$ and 7 post-operatively. RESULTS: On the day of surgery, the IMP procedure gave the lowest median VAS for all four PROM parameters. After a week, OFD still had a significantly higher VAS for swelling, pain and bruising. Patients who underwent procedures lasting more than $60 \mathrm{~min}$. had higher VAS for all parameters except bleeding. After considering other important confounders, type of surgery procedure was no longer associated with the VAS score for any of the parameters. Time after surgery, male gender and shorter surgery duration reduced post-operative VAS for one or more of the parameters. Longer surgeon experience helps reduce VAS scores only for bleeding. Prevalence for tenderness to palpation was $11.6 \%, 8.9 \%$ and $12.2 \%$ for IMP, CL and OFD, respectively, 1-week post-operatively. Swelling and suppuration occurred rarely. CONCLUSIONS: The median VAS scores for all PROM parameters were generally low and reduced to near zero over a week following all three surgical procedures tested. Time after surgery and shorter surgery duration were associated with lower VAS scores in all the PROM parameters in this cohort of patients. Surgery type was not associated significantly with VAS after adjustment with other important confounders. Low prevalences of post-surgical complications were reported.
\end{abstract}

DOI: https://doi.org/10.1111/jcpe.12248

Posted at the Zurich Open Repository and Archive, University of Zurich

ZORA URL: https://doi.org/10.5167/uzh-108003

Journal Article

Accepted Version

Originally published at:

Tan, Wah Ching; Krishnaswamy, Gita; Ong, Marianne M A; Lang, Niklaus P (2014). Patient-reported outcome measures after routine periodontal and implant surgical procedures. Journal of Clinical Periodontology, 41(6):618624.

DOI: https://doi.org/10.1111/jcpe.12248 


\section{A pilot study on patient reported outcome measures after routine periodontal and implant surgical procedures.}

Wah Ching Tan ${ }^{1}$, Gita Krishnaswamy ${ }^{2}$, Marianne MA Ong ${ }^{1}$, Niklaus P Lang ${ }^{1,3,4}$

1) National Dental Centre Singapore, Singapore

2) Centre for Quantitative Medicine, Duke-NUS Graduate Medical School

3) University of Zurich, Switzerland

4) The University of Hong Kong, Hong Kong, SAR PR China

Running head: PROMs after surgical procedures

Key words: Patient Reported Outcome Measures, periodontal, implant, surgery, crown lengthening

Corresponding address:

Dr. Wah Ching Tan, BDS, MDS, Dr.med.dent.

Consultant, National Dental Centre Singapore

5 Second Hospital Avenue

Singapore 168938

drwahching@yahoo.com.sg

Phone: +6598553559

The study was funded by means of the National Dental Centre, Singapore.

The authors declare no conflict of interest. 


\section{Abstract}

Objectives: -To compare patient reported outcome measures (PROMs) after different dental surgical procedures over a 1-week post-surgical period and in relation to duration of the surgery, and periosteal releasing incisions.

-To evaluate the prevalence of post-surgical complications.

Material \& methods: 468 healthy dental patients requiring surgeries, such as crown lengthening ( $C L$ ), open flap debridement (OFD), and implant installation (IMP) in the National Dental Centre, Singapore (2009-2011) were consecutively recruited. PROMs on bleeding, swelling, pain and bruising were obtained using Visual Analogue Scales (VAS) on days 0, 3, 5 and 7 postoperatively. Results: On the day of surgery, the IMP procedure gave the lowest median VAS for all four PROM parameters. After a week, OFD still had a significantly higher VAS for swelling, pain and bruising. Patients who underwent procedures lasting more than 60 minutes had higher VAS for all parameters except bleeding. After considering other important confounders, type of surgery procedure was no longer associated with the VAS score for any of the parameters. Time after surgery, male gender, and shorter surgery duration reduced post-operative VAS for one or more of the parameters. Longer surgeon experience helps reduce VAS scores only for bleeding. Prevalence for tenderness to palpation were $11.6 \%, 8.9 \%, 12.2 \%$ for IMP, CL, OFD, respectively 1-week post-operatively. Swelling and suppuration occurred rarely.

Conclusions: The median VAS scores for all PROM parameters were generally low and reduced to near zero over a week following all three surgical procedures tested. Time after surgery and shorter surgery duration were associated with lower VAS scores in all the PROM parameters in this cohort of patients. Surgery type was not associated significantly with VAS after adjustment with other important confounders. Low prevalences of postsurgical complications were reported. 


\section{Clinical Relevance}

Scientific rationale for the study: Patient Reported outcome measures (PROMs) after surgical procedures are usually neglected and are rarely reported and yet, they constitute an important and relevant clinical aspect of therapy.

Principle findings: During the first week of healing, following dental surgical procedures, PROMs for bleeding, swelling, pain and bruising are generally perceived as relatively low. Visual Analogue Scale scores for these outcomes were lower for implant installation than for periodontal surgical interventions. Male gender, and shorter surgery duration reduced post-operative VAS for one or more of the PROM parameters.

Practical implications: Periodontal surgeries, in general, yield low morbidity and minor perceived bleeding, swelling, pain and bruising and hence, should not create anxiety for the patient. Reduction in the time spent in a surgery resulted in reduced post-operative VAS scores. 


\section{Introduction}

Periodontal and implant surgeries belong to routine treatment in current clinical practice. With advancements in science, biomaterials and surgical techniques, these procedures may be carried out predictably by well-trained professionals. With regards to outcomes, many studies reported low rates of post-operative infection and complications following periodontal and implant surgeries (Powell et al. 2005). However, little is known on the patients' perception after oral surgical procedures. In few studies reporting on patients' perception, visual analogue scales (VAS) were used as assessment tools. Moreover, VAS were used to assess patients' dental anxiety before and after extractions (Luyk et al.1988). More recently (Fardal \& McCulloch 2012), VAS were used to assess patients' anxiety and pain perception in a private periodontal practice setting. Furthermore, VAS scores appear to be valid tools that were used to assess dental pain perception (Seymour et al. 1983). There is, however, few studies using this tool to assess other aspects of postsurgery sequelae, such as swelling, bleeding and bruising. Currently, no studies have compared patient reported outcome measures (PROMs) of postsurgical sequelae of periodontal and implant surgeries. PROMs involving patients' perception of bleeding, pain, swelling, bruising during the first week post-operative healing period may be important in setting a patient's expectations.

Various types of periodontal surgeries, such as soft tissue grafts and osseous surgery have been reported to produce more discomfort than non-surgical therapy (Matthews \& McCulloch 1993). Canakçi \& Canakçi (2007) also reported significantly higher post-surgical pain for gingivectomies and osseous procedures than for scaling and root planing and modified Widman flap (open flap debridement) in periodontal patients.Factors like duration of surgery and use of periosteal releasing incision may affect the patients' postsurgical perception. 
Therefore, the aim of this study was to examine PROMs for types of dental surgeries during the first week post-surgical period and the effect of other confounding factors on PROMs. The prevalences of post-surgical complications were also analyzed. 


\section{Material \& methods}

Subject population \& Ethics approval

During the period 2009-2011, the Periodontics Unit of the National Dental Centre, Singapore conducted a clinical audit on PROMs in relation to dental surgeries as part of the Centre's outcome measures data collection for the Centre. Patients scheduled for periodontal surgeries were informed data related to their surgery and their perceptions after the surgery would be collected for audit and study purposes.

These surgical procedures were either crown lengthening $(C L)$, open flap debridement (OFD) or straight forward implant installation surgery (IMP). The relevant informed consent for the surgeries was obtained by the patients' attending surgeons. There was no sample size calculation as this study was part of an observational clinical audit of outcome measures in the centre. The protocol was submitted to and approved by the SingHealth Centralised Institutional Review Board for IRB exemption.

\section{Surgical procedures}

The patients proceeded with their scheduled surgeries following initial debridement of the oral cavity. Sterile instruments and aseptic techniques were employed for all surgeries, with adequate amount of anaesthesia provided at the operation site, and the surgeons-in-charge filled in a data collection form on the type of surgery performed, the duration of surgery and whether or not periosteal releasing incisions were used. Eight periodontists had performed any of the three surgical procedures ( $C L$, OFD, IMP). The surgeons involved had at least two years of experience as specialist periodontists and were well-trained to perform both periodontal and implant surgeries. The patients were given standard post-surgical instructions including achieving haemostasis with a gauze over the surgical site for 30 
minutes. All patients were prescribed analgesics after their surgeries based on their surgeons' preference.

The patients charted their perceptions on bleeding, swelling, pain, and bruising over the first week healing period, on days 0 (at night prior to sleeping), 3, 5, 7 using a VAS with equal units from 0 to 10 (on a line of 10 centimetres) with 0 designated as no bleeding/ swelling/ pain/ bruising, to 10 for severe excruciating bleeding/ swelling/ pain/ bruising. The patients were given instructions on charting of the VAS by their respective surgeons.

On the day of suture removal (Day 7), patients were examined clinically by their surgeon for post-surgical complications (tenderness on palpation, swelling, suppuration).

Outcome variables

- The primary outcome variables were PROMs using a VAS score on bleeding, swelling, pain, and bruising.

- The secondary outcome variables were the prevalences of postsurgical complications, namely tenderness on palpation, swelling, suppuration on Day 7.

\section{Statistical analysis}

This was an observational clinical audit study and surgical procedures were already scheduled for the patients prior to their entry into the study. Hence no sample size calculations were done. The samples by surgical procedure were not balanced but were large enough for statistical analysis. Collated data from individual patient data collection forms that had been de-identified were entered into a database in an Excel Spreadsheet. All analyses were performed using SAS statistical software, Version 9.2.

The outcome variable, the VAS score was skewed in its distribution and failed the normality test. Non-parametric Kruskal-Wallis and Wilcoxon-MannWhitney tests were used in bivariate analysis that allowed groups to be 
compared for differences in medians and spread of the VAS score without making the assumption of normal distribution. Post-operative VAS for each PROM parameter, considering multiple confounders such as gender, procedure type, surgery duration, surgeon experience, use of painkillers and periosteal releasing incisions was analyzed using a linear random-effect mixed model. All results were interpreted at a level of significance of 0.05 .

\section{Results}

Demographics and Surgical factors (Table 1)

468 healthy patients requiring periodontal or implant surgeries were consecutively recruited. Overall, $42 \%$ of the subjects were males and a large majority $(85 \%)$ were Chinese. The surgeries performed included crown lengthening ( $C L, n=259)$, straight forward implant installation surgery (IMP, $\mathrm{n}=115$ ) and open flap debridement (OFD, $\mathrm{n}=94$ ). $70 \%$ of the surgeries were less than 60 minutes duration and $56 \%$ were performed by surgeons with more than 5 years' experience.

\section{Patient reported outcome measures (PROMs)}

PROMs on parameters such as bleeding, swelling, pain, and bruising were collected at Days $0,3,5$ and 7 . Compliance of the patients with the questionnaire immediately after the surgery was $100 \%$.

\section{Procedure type (Table 2)}

On the day of surgery, IMP had the lowest median VAS scores for all PROM parameters. . Median bleeding VAS scores decreased to almost zero for all procedures after Day 0 (Figure 1). The decrease in VAS scores for swelling, pain and bruising over the 7 days was the slowest for OFD (Figure 2-4). At the end of the week, OFD still had a significantly higher VAS for those parameters. 
Duration of surgery (Table 3)The duration of surgery was stratified to less than 60 minutes or equal to or more than 60 minutes. VAS scores for bleeding were not significantly different for the surgery durations. Surgeries lasting 60 minutes or longer had statistically higher VAS scores for swelling and bruising for all the days. Pain had significantly higher VAS scores for longer surgeries after the day of surgery. The use of periosteal releasing incisionAfter adjusting for the confounders, the use of periosteal releasing incision resulted in significantly higher VAS scores for swelling even though there was $50 \%$ of missing data from the collection forms for this field (Table 4).

Mixed model to examine adjusted post-operative VAS (Table 4)

The effect of surgical procedure on VAS for bleeding, swelling, pain and bruising, adjusted for multiple confounders including gender, ethnicity, surgery duration, surgeon experience, use of analgesics and use of periosteal releasing incisions was analyzed using a linear mixed random-effects model. In the multivariable model with all the confounders, surgery duration of less than 60 minutes and post-operative days significantly decreased VAS scores for all the PROM parameters. In addition VAS score for swelling was also significantly reduced for males and no periosteal releasing incisions. Longer surgeon experience only reduced VAS score for bleeding. Use of analgesics was not associated with the VAS score. Type of surgery procedure after adjusting for all the confounders was no longer associated with VAS score.

\section{Post-surgical complications}

At the 1-week clinical re-evaluation, flap dehiscences were noted in $13.7 \%$ of IMP, $8.0 \%$ of $\mathrm{CL}$, and $14.6 \%$ of OFD cases. Prevalence of tenderness to palpation was $11.6 \%$ for IMP, $8.9 \%$ for $\mathrm{CL}$, and $12.2 \%$ for OFD. The prevalence of swelling and suppuration was $<5 \%$ in the 3 types of surgical procedures. 


\section{Discussion}

This study has demonstrated that the patient's perception for bleeding, swelling, pain and bruising during the first week of healing following various oral surgical procedures was modest and generally within the evaluation of "well tolerated". This is in agreement with studies on "straight forward" implant installation (Hashem et al. 2006, Al-Khabbaz et al. 2007) and on periodontal flap surgical procedures (Fardal \& McCulloch 2012). However, the results do not concur with another more recent report on patient's perception of postoperative pain after periodontal surgery (Canakçi \& Canakçi 2007) in which VAS scores for pain were slightly higher. The reason for this discrepancy is not completely understood. Cultural differences in pain perception may - at least in part - contribute to the difference in addition to the type of surgeries they had performed: the modified Widman flap, flap with osseous resection and gingivectomy. In our study, the surgical procedures were CL, OFD and IMP. CL encompassed either soft tissue or hard tissue or a combination of both soft and hard tissue reduction. Data collected from this observational clinical audit study did not allow us to differentiate between the types of CL procedure performed.

Moreover, the present study has identified significant differences in PROMs between IMP and OFD and CL procedures, the first yielding lower VAS scores. This, in turn, suggests that IMP represents a surgical procedure with minor morbidity compared to the periodontal surgical procedures ( $C L$ and OFD). This is in contrast with a comparative study on the patients' perception of periodontal and implant surgeries (Fardal \& McCulloch 2012). These authors reported no significant differences between patients' perception of periodontal and implant surgeries, although there was a significantly higher mean anxiety score for implant surgeries compared to periodontal surgeries. It was also found that patients' with high anxiety score experienced more discomfort during the surgery. In the present study, patient's anxiety was not evaluated. 
VAS scores were used in the present as well as in the study mentioned earlier as it is a frequently used tool for assessing patients' perceptions (Seymour et al. 1983). It is easy to administer and is reproducible.

In a study on pain and anxiety following implant surgeries (Hashem et al. 2006), an average pain score of 24/100 on day 1 after the surgery was reported. Mean pain scores were highest also at 24 hours after surgery, with a score of 20.1/100 in another study (Al-Khabbaz et al. 2007). In the present study, the highest median pain scores were recorded on the day of the surgery itself. The median pain score of $10 / 100$ was lower than that mentioned in the two former studies. This difference may be explained by the fact that in the present study, PROMs were recorded on the day of surgery and on Day 3 with no registrations in between. Hence, it may be anticipated that pain sensations may still increase from the day of surgery to the first and second postoperative day.

Moreover, the present study has identified two aspects that significantly influenced the PROMs during the first week of healing, the duration of the surgical procedure and the application of periosteal releasing incisions. Both aspects negatively influenced the PROMs. Longer surgical time of 60 minutes or more resulted in statistically higher VAS scores for swelling and bruising on the day of surgery itself, when compared to those who had undergone procedures done within 60 minutes. At 1-week post-surgery, patients who had undergone a procedure that took 60 minutes or longer had statistically higher VAS scores for swelling, pain and bruising when compared to those who had undergone procedures done within 60 minutes. The duration of the surgical procedure has been reported to be highly related to pain and swelling post-surgery following gingival augmentation procedures (Griffin et al. 2006).

Patients with periosteal releasing incisions reported significantly higher median VAS scores for swelling when compared to those without, on the day of the surgery. It is of interest to note that this difference was found to be 
significant even though a large proportion of this data was missing from the data collection forms.

Limitations of this study are as follows:

1) The data was obtained from a clinical audit, thus the study groups were not balanced in numbers and detailed information on location and number of teeth operated on are lacking. Other information with regards to patients' age, whether osseous surgery was done and type of $C L$ surgery were unavailable. In addition, data for some variables was inadvertently missing due to some surgeons failing to fill in some data fields (use of periosteal releasing incision $-50 \%$ missing data, all others $<3 \%$ ) in the data collection forms.

2) Eight surgeons performed the surgeries and assessed their own patients for post-surgical complications on the day of suture removal. In addition, type of analgesic prescription was not standardized among surgeons.

3) There are other parameters of the patients' experience before and after surgery that we were unable to assess in this study. These include patients' pre-surgical anxiety scores, overall satisfaction of the surgery outcome, root hypersensitivity, chewing functions after the surgery and oral health related quality of life measurements. These should be considered in future studies on patients' perception post-dental surgery.

Despite the above mentioned short-comings, this pilot study has merit with regards to reporting on PROMs after surgical procedures as the data was collected from a relatively large number of patients and the 3 types of surgical procedures were performed by specialists.

At the National Dental Centre of Singapore, all the procedures were performed by board certified periodontists who had at least two years of experience as a practicing specialist. It should also be noted that this study was carried out in an institution periodontal specialist practice and the results may not necessarily apply to a different setting.

Anecdotal evidence based on clinical experience and observation seem to associate female patients, longer duration of surgical procedures and use of periosteal releasing incisions with patients experiencing more discomfort after oral surgical procedures. The results of this study, although observational in 
nature, appear to provide some preliminary support to some of these factors that may have an influence on an individual's perception of PROMs after oral surgical procedures.

Future studies investigating PROMs after oral surgical procedures should be designed with the view to balance the types of surgery in groups to be compared, minimising operator variability and the standardisation of analgesic prescription.

Conclusion

Bearing in mind the stated limitations of this study, we conclude that the median VAS scores for all PROM parameters (bleeding, swelling, pain, and bruising) were generally low and reduced to near zero over a week following implant and periodontal surgical procedures in this cohort of patients. Time after surgery and shorter surgery duration were associated with lower VAS scores in all the PROM parameters in this cohort of patients. Surgery type was not associated significantly with VAS after adjustment with other important confounders.

Low prevalences of post-surgical complications were reported at the 1-week post-surgical review.

\section{Acknowledgements}

The co-operation of the following staff of the National Dental Centre Singapore is highly appreciated: Drs. Chee Hoe Kit, , Koh Chu Guan, Mervyn $\mathrm{Ng}$, Tan Ching Ching, Wong Li Beng, Alvin Yeo. 


\section{References}

Al-Khabbaz, A.K., Griffin, T.J. \& Al-Shammari, K.F. (2007) Assessment of pain associated with the surgical placement of dental implants. Journal Periodontology 78: 239-246.

Canakçi, C.F. \& Canakçi, V. (2007) Pain experienced by patients undergoing different periodontal therapies. Journal American Dental Association 138:1563-1573.

Fardal, Ø. \& McCulloch, C.A. (2012) Impact of anxiety on pain perception associated with periodontal and implant surgery in a private practice. Journal Periodontology 83: 1079-1085.

Griffin T.J., Cheung, W.S., Zavras, Al., Damoulis, P.D. (2006) Postoperative complications following gingival augmentation procedures. Journal Periodontology 77: 2070-2079.

Hashem, A.A., Claffey, N.M. \& O'Connell, B. (2006) Pain and anxiety following the placement of dental implants. International Journal Oral Maxillofacial Implants 21: 943-950.

Luyk, N.H., Beck, F.M. \& Weaver, J.M. (1988) A visual analogue scale in the assessment of dental anxiety. Anesth Prog 35: 121-123.

Matthews, D. C. \& McCulloch, C.A.G. (1993) Evaluation patient perceptions on short-term outcomes of periodontal treatment. A comparison of surgical and non-surgical therapy. Journal Periodontology 64: 990-997.

Powell, C.A., Mealey, B.L., Deas, D.E., McDonnell, H.T. \& Moritz, A.J. (2005) Post-surgical infections: prevalence associated with various periodontal surgical procedures. Journal Periodontology 76: 329-333 
Seymour, R., Charlton, J. \& Phillips, M. (1983) An evaluation of dental pain using visual analogue scales and the McGill pain questionnaire. Journal Oral Maxillofacial Surgery 41: 643-648.

\section{Legends}

Figure 1. VAS Scores for bleeding by surgical procedures (Implant installation, Crown lengthening, Open Flap Debridement) and by day.

Figure 2. VAS Scores for swelling by surgical procedures (Implant installation, Crown lengthening, Open Flap Debridement) and by day.

Figure 3. VAS Scores for pain by surgical procedures (Implant installation, Crown lengthening, Open Flap Debridement) and by day.

Figure 4. VAS Scores for bruising by surgical procedures (Implant installation, Crown lengthening, Open Flap Debridement) and by day. 